\title{
A Cross Sectional Study of Fasting Serum Magnesium Levels In Patients with Type 2 Diabetes Mellitus Attending Chhatrapati Shivaji Subharti Hospital
}

\author{
Singhal S, Kumar S
}

\section{Introduction}

Diabetes is a group of metabolic disease characterized by hyperglycemia resulting from defects in insulin secretion, insulin action, or both .The chronic hyperglycemia of diabetes is associated with long term damage, dysfunction, and failure of different organs, especially the eyes, kidneys, nerves, heart and blood vessels.Several pathogenic processes are involved in the development of diabetes These ranges from autoimmune destruction of the beta cells of the pancreas with consequent insulin deficiency to abnormalities that results in resistance to insulin action.(1)

Magnesium is the second most abundant intercellular cation after potassium present in living cells.Of the 21-28 gram of magnesium present in adult human body,99\% is distributed in the intercellular compartment,and only $1 \%$ in the extracellular fluid.Magnesium is subdivided into three major compartments of the body;about $65 \%$ in the mineral phase of skeleton,34\% in the intercellular space and only $1 \%$ in the extracellular fluid.(2)Serum concentration of magnesium ranges from 1.7-2.4mg/dl(0.7-1 mmol/l) The plasma concentration in healthy adults remains remarkably constant, whereas $25-39 \%$ of diabetics have low concentrations of serum magnesium .(3)Studies have reported serious complications of diabetes leading to hypomagnesemia which depends on various factor, such as age ,sex ,duration of diabetes, etc.(4) The present study was undertaken with an aim to estimate prevalence of hypomagnesemia in patient with type II diabetes mellitus and to correlate the serum magnesium concentrations with age ,sex ,duration of diabetes, etc.

\section{Aims And Objectives}

To estimate fasting magnesium levels in patients with type 2 Diabetes mellitus and correlate serum magnesium concentrations with Microvascular and Macrovascular complications of type II diabetes mellitus i.e. Retinopathy, Nephropathy and Neuropathy.

Type of study : Cross sectional study

\section{Material and Methods}

Place of Study : chhatrapati shivaji subharti hospital

Subharti medical college, meerut, u.p.

The results are subjected to appropriate statistical analysis.Before starting the study, all patients was informed about the study and consent was duly taken.All patients were undergone detailed history and clinical examinations to fulfill the inclusion and exclusion criteria.

Sample size: 100 patients

Inclusion Criteria

All diagnosed cases of type II diabetes mellitus attended Chhatrapati Shivaji Subharti Hospital, Meerut.

\section{Exclusion Criteria}

1. Patients with chronic renal failure.

2. Acute myocardial infarction in last 6 months.

3. Patients on medicines which cause alteration in serum magnesium level e.g.Aminoglycosides, Amphotericin

B,Cyclosporine, Digoxin, Diuretics (loop, thiazides, osmotic).

4. Patients receiving magnesium supplements or magnesium containing antacids.

5. Patients with history of chronic alcohol abuse.

6. Patients with malabsorption or chronic diarrhea.

7. All pregnant women.

8.Patients taking antiepileptic drugs. 


\section{History and Physical examination}

\section{Methodology:}

All patients with type II diabetes mellitus who meet the inclusion criteria were evaluated, detailed history including age, gender, duration of diabetes, symptoms suggestive of diabetic neuropathy, retinopathy, nephropathy, associated disease such as hypertension and ischemic heart disease and current use of medication, including anti diabetic drugs was obtained by a standard interview questionnaire, followed by

- Physical and neurological examination,

- Twelve lead electrocardiography,

- Fundus examination of both eyes done by direct ophthalmoscopic examination to confirm diabetic retinopathy,

- Monofilament examination

- Nerve conduction study to confirm the presence of diabetic neuropathy.

\section{Investigation}

1. Serum magnesium level

2. $\mathrm{HbA1c}$

3. Fasting blood sugar

4. Serum Urea and creatinine level

5.24 hour urinary albumin/Spot urine protein

\section{Results}

Study group comprised of 100 cases with type 2 diabetes mellitus.These cases were further grouped with regards their age,gender,glycemic control,and presence or absence of diabetic complications(diabetic retinopathy,diabetic neuropathy and diabetic nephropathy) Prevalence of hypomagnesemia

Out of total 100 cases in the study, 34 were found to have hypoglycemia. Prevalence of hypomagnesemia according to age distribution Majority of the cases $(n=52)$ were in the age group of 51-60 years. Maximum prevalence $(50 \%)$ of hypomagnesemia with $\mathrm{p}$ value 0.21 was found in age group of more than 70 years and minimum prevalence( $10 \%$ ) in less than 50 years.

Table no.1 Prevalence of hypomagnesemia according to age distribution

\begin{tabular}{|l|l|l|l|l|}
\hline \multirow{2}{*}{ Age in years } & \multirow{2}{*}{ No.of Cases } & \multicolumn{2}{|l|}{ Prevalence of Hypomagnesemia } & \multirow{2}{*}{$\mathrm{p}$ Value } \\
\cline { 3 - 4 } & & Frequency & $\%$ & \\
\hline $41-50$ & 10 & 1 & $10 \%$ & \multirow{2}{*}{0.21} \\
\hline $51-60$ & 52 & 18 & $34.6 \%$ & \\
\hline $61-70$ & 32 & 12 & $37.5 \%$ & \\
\hline$>70$ & 6 & 3 & $50 \%$ & \\
\hline Total & 100 & 34 & 34 & \\
\hline
\end{tabular}

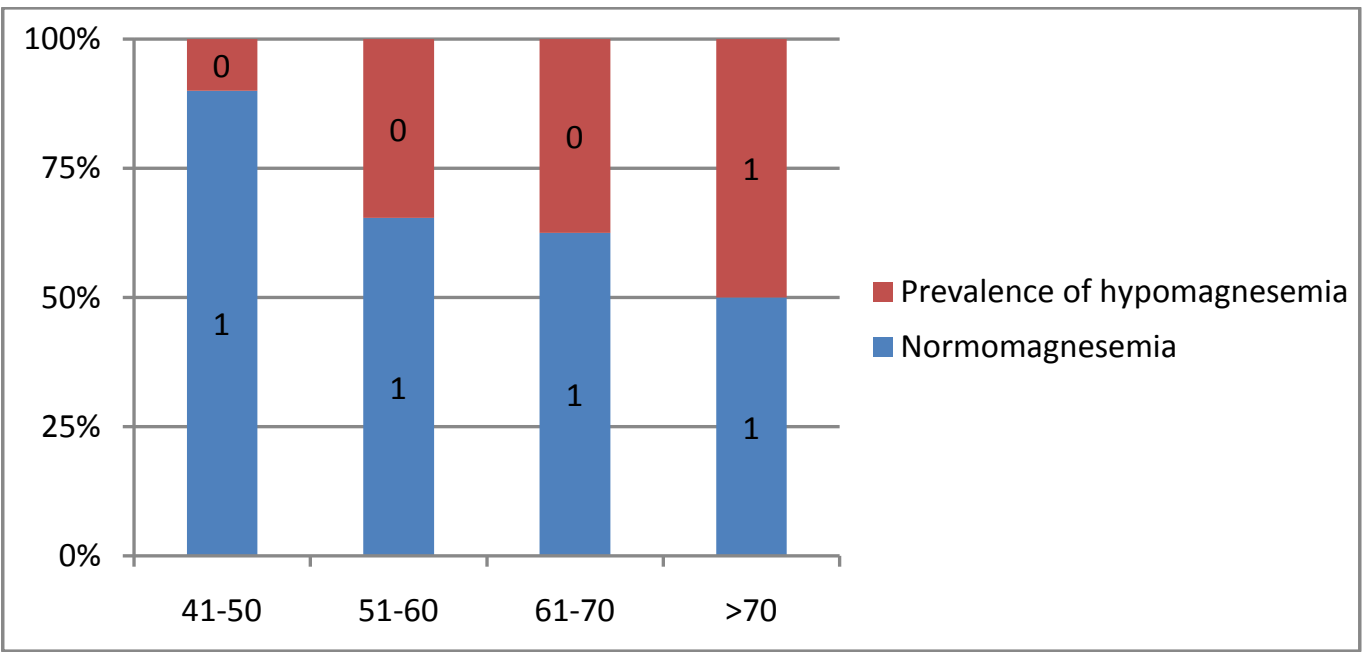




\section{Prevalence Of Hypomagnesesemia According To Sex}

Out of the 100 cases ,75(75\%) were males and 25(25\%) were females.Twenty four (32\%) of the males and ten $(40 \%)$ of the females were found to have hypomagnesemia.There was no statistically correlation between any of the gender and hypomagnesemia.

Prevalence of Hypomagnesemia according to Sex
\begin{tabular}{|l|l|l|l|l|}
\hline \multirow{2}{*}{ Sex } & No.of cases & Prevalence of Hypomagnesemia & \multirow{2}{*}{ v Value } \\
\cline { 2 - 4 } & & Frequency & $\%$ & \multirow{3}{*}{0.27} \\
\hline Male & 75 & 24 & $32 \%$ & \\
\hline Female & 25 & 10 & $40 \%$ & \\
\hline Total & 100 & 34 & $34 \%$ & \\
\hline
\end{tabular}

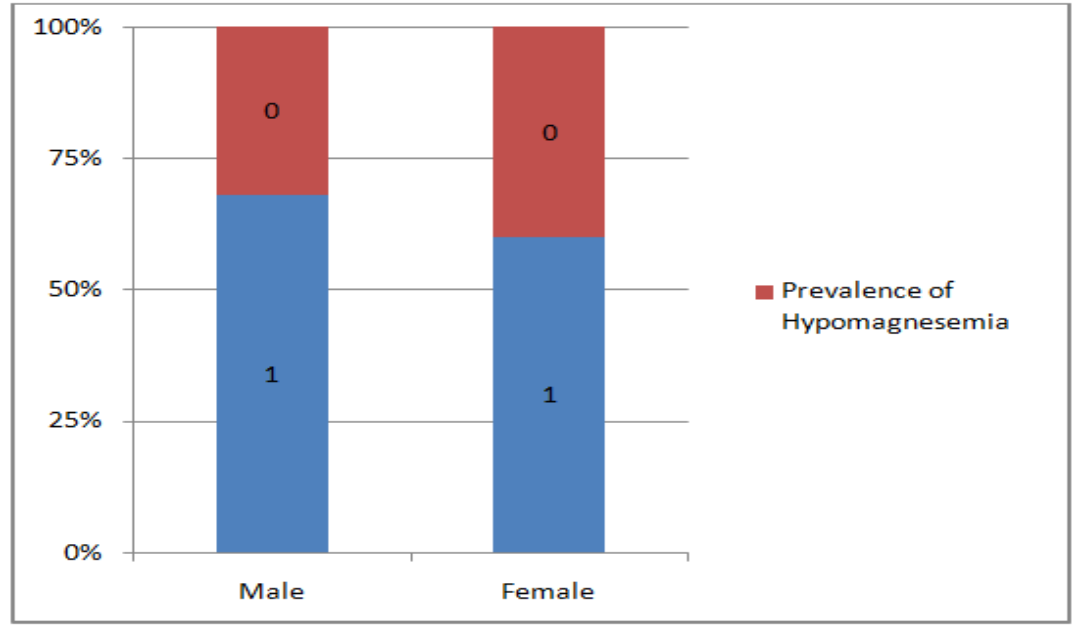

\section{Prevalence Of Hypomagnesesemia According To Duration Of Diabetes}

Out of all cases, the prevalence of hypomagnesemia was found to be highest $(66.6 \%)$ in the group having duration of diabetes for more than 20 years with p value 0.87 . The lowest prevalence $(30.6 \%)$ of hypomagnesemia was found in the group with duration of diabetes less than 5 years with p value 0.87 , however majority of the cases $(n=62)$ belongs to the group.Prevalence of Hypomagnesemia according to duration of diabetes

\begin{tabular}{|l|l|l|l|l|}
\hline \multirow{2}{*}{$\begin{array}{l}\text { Duration of } \\
\text { diabetes in years }\end{array}$} & No.of Cases & \multicolumn{2}{|l|}{ Prevalence of Hypomagnesemia } & \multirow{2}{*}{ v Value } \\
\cline { 3 - 4 } & & Frequency & $\%$ & \\
\hline $0-5$ & 62 & 19 & $30.6 \%$ & \multirow{3}{*}{0.87} \\
\hline $6-10$ & 20 & 7 & $35.0 \%$ & \\
\hline $11-15$ & 10 & 4 & $40.0 \%$ & \\
\hline $16-20$ & 5 & 2 & $40.0 \%$ & \\
\hline$>20$ & 3 & 2 & $66.6 \%$ & $34 \%$ \\
\hline Total & 100 & 34 & & \\
& & & & \\
\end{tabular}

Prevalence of Hypomagnesemia according to duration of diabetes

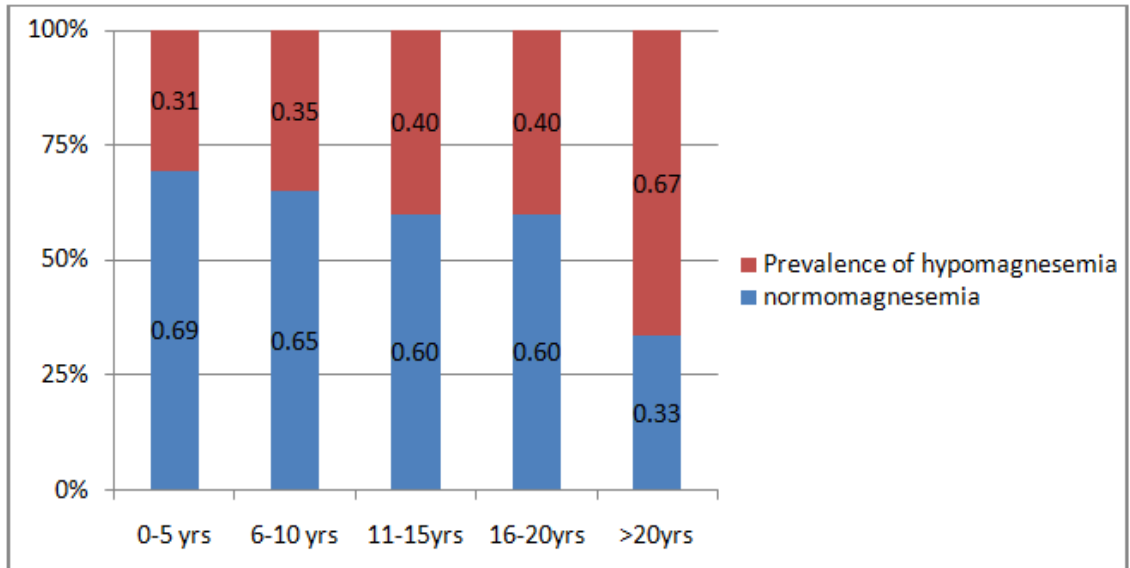




\section{Prevalence of hypomagnesemia according to $\mathrm{HbA1C}$}

Out of the 100 cases, ,the prevalence of hypomagnesemia was found to be highest $(40.7 \%)$ in the group with $\mathrm{HbA1C}$ more than $10 \%$ with $\mathrm{p}$ value 0.79 .The lowest $(25 \%$ )prevalence of hypomagnesemia was in the group with $\mathrm{HbA} 1 \mathrm{C}$ less than with p value 0.79 .

Prevalence of Hypomagnesemia according to HbA1c

\begin{tabular}{|l|l|l|l|l|}
\hline \multirow{2}{*}{ HbA1c(\%) } & No.of Cases & \multicolumn{2}{|l|}{ Prevalence of Hypomagnesemia } & \multirow{2}{*}{ p Value } \\
\cline { 3 - 4 } & & Frequency & $\%$ & \\
\hline$<6$ & 4 & 1 & $25.0 \%$ & \multirow{2}{*}{0.79} \\
\hline $6-7$ & 29 & 10 & $34.4 \%$ & \\
\hline $7-8$ & 19 & 6 & $31.6 \%$ & \\
\hline $8-9$ & 11 & 3 & $27.7 \%$ & $30.0 \%$ \\
\hline $9-10$ & 10 & 3 & $40.7 \%$ & \\
\hline$>10$ & 27 & 11 & \\
\hline
\end{tabular}

Prevalence of Hypomagnesemia according to HbA1c

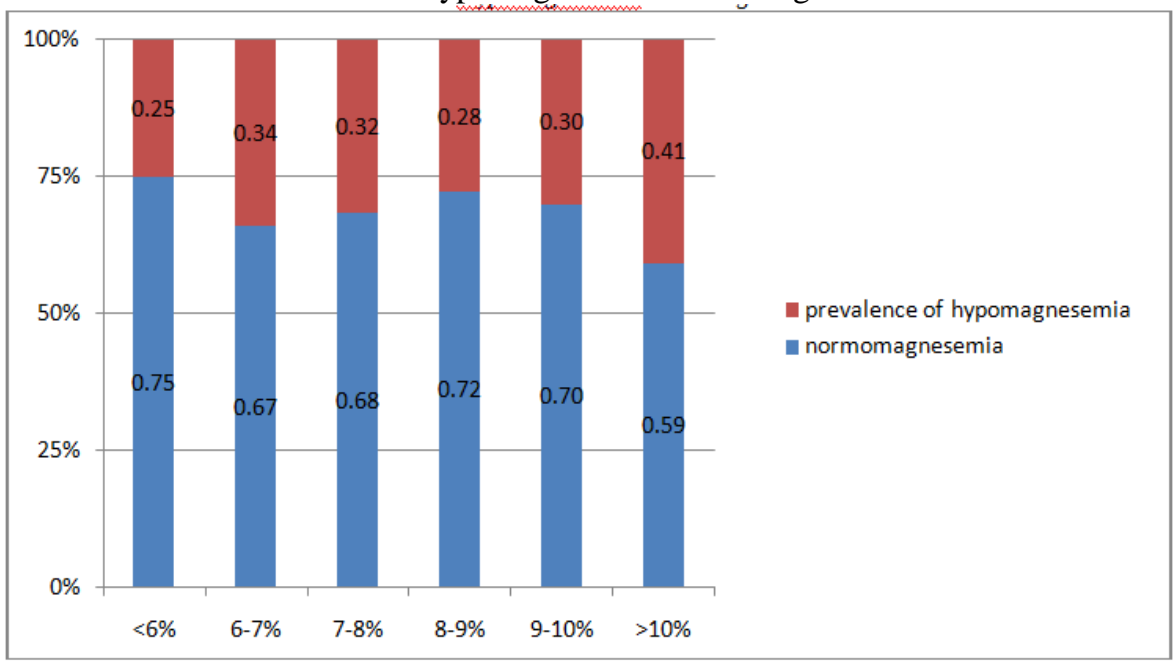

\section{Prevalence of hypomagnesemia in Diabetic Retinopathy patients}

Out of the 100 cases , 26 cases had non proliferative diabetic retinopathy, within which 15(57.7\%) had hypomagnesemia. 6 cases had proliferative diabetic retinopathy and among them $3(50 \%)$ had hypomagnesemia. Thus among the group with retinopathy(non proliferative and proliferative) $56.2 \%$ of the patient had hypomagnesemia while only $23.5 \%$ of the cases without retinopathy had the same. This difference was statistically significant with p valueless than $0.05 \%$

\begin{tabular}{|l|l|l|l|l|l|}
\hline \multicolumn{2}{|l|}{ Retinopathy } & \multirow{2}{*}{ Total patients } & \multicolumn{2}{l}{$\begin{array}{l}\text { Prevalence } \\
\text { Hypomagnesemia }\end{array}$} & \multirow{2}{*}{ P Value } \\
\cline { 3 - 5 } & & Frequency & $\%$ & \\
\hline \multirow{3}{*}{ Retinopathy } & NPDR(nonproliferative) & 26 & 15 & $57.7 \%$ & \multirow{2}{*}{0.002} \\
\cline { 2 - 5 } & PDR(Proliferative) & 6 & 3 & $50 \%$ & $56.2 \%$ \\
\cline { 2 - 5 } & Total & 32 & 18 & $23.5 \%$ & \\
\hline No Retinopathy & 68 & 16 & & \\
\end{tabular}

Prevalence of hypomagnesemia in Diabetic Retinopathy patients

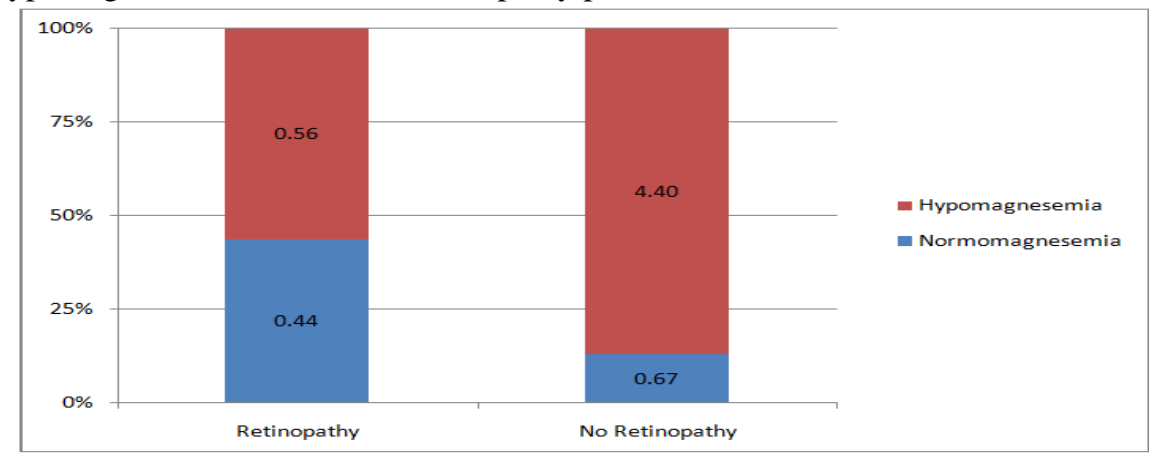




\section{Prevalence of hypomagnesemia in Diabetic Nephropathy Patients}

Out of the 100 cases, 14 were found to have nephropathy while 86 did not have the same.Of the 14 cases who had nephropathy 11 were diagnosed with microalbuminuria,36.6\% of this group were diagnosed with hypomagnesemia.Three cases from the nephropathy group were diagnosed with macroalbuminuria, $66.6 \%$ of the cases from this group had hypomagnesemia.Thus the prevalence of hypomagnesemia in cases with nephropathy was found to be $42.8 \%$ as opposed to $32.5 \%$ of the group with no nephropathy which was statistically significant.

\begin{tabular}{|c|c|c|c|c|c|}
\hline \multirow{2}{*}{\multicolumn{2}{|c|}{ Nephropathy }} & \multirow{2}{*}{$\begin{array}{l}\text { Total } \\
\text { Patien } \\
\text { ts }\end{array}$} & \multicolumn{2}{|c|}{$\begin{array}{l}\text { Prevalence of } \\
\text { Hypomagnesemia }\end{array}$} & \multirow[t]{2}{*}{ P Value } \\
\hline & & & Frequency & $\%$ & \\
\hline \multirow[t]{3}{*}{ Nephropathy } & Microalbuminuria & 11 & 4 & $36.6 \%$ & \multirow{4}{*}{0.002} \\
\hline & Macroalbuminuria & 3 & 2 & $66.6 \%$ & \\
\hline & Total & 14 & 6 & $42.8 \%$ & \\
\hline \multicolumn{2}{|c|}{ No Nephropathy } & 86 & 28 & $32.5 \%$ & \\
\hline
\end{tabular}

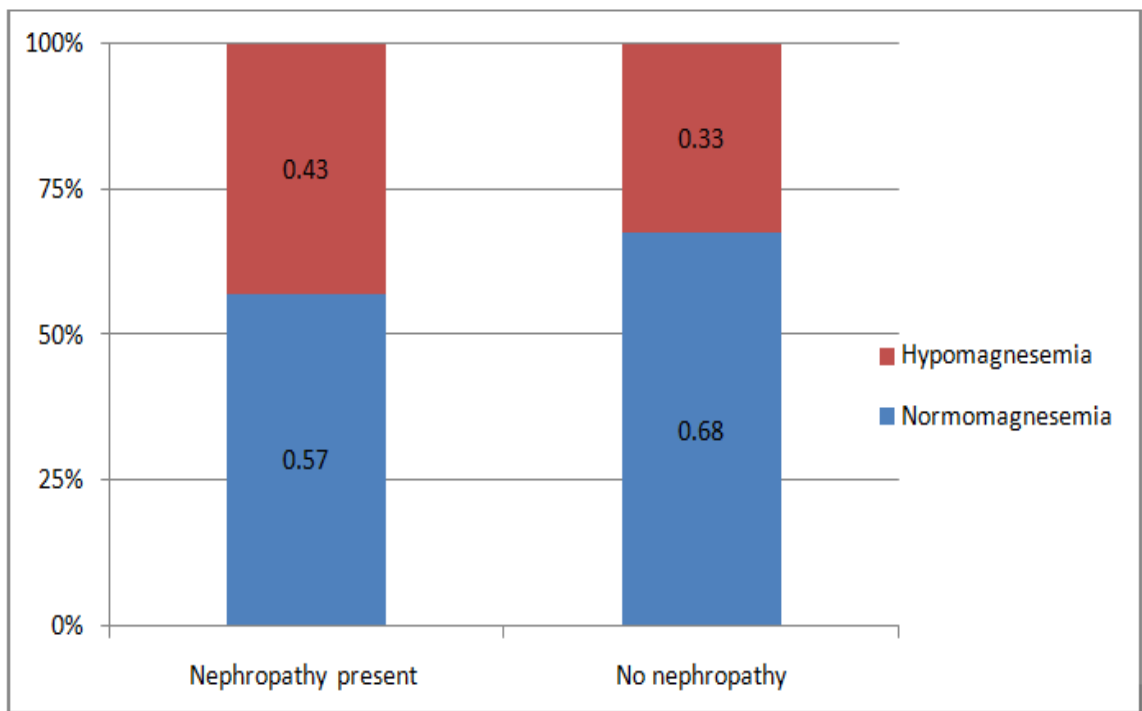

Prevalence of hypomagnesemia in Diabetic Neuropathy Patients

Out of the 100 cases, 17 (17\%)cases had diabetic neuropathy while 83(83\%) did not have diabetic neuropathy.7 cases $(41.1 \%)$ of the cases who had neuropathy also had hypomagnesemia, while 27 cases $(32.5 \%)$ of the cases with no neuropathy were found to have hypomagnesemia.

\begin{tabular}{|l|l|l|l|l|}
\hline Neuropathy & \multirow{2}{*}{ No. of cases } & \multicolumn{2}{|l|}{ Prevalence of hypomagnesemia } & \multirow{2}{*}{ P value } \\
\cline { 3 - 4 } & & Frequency & $\%$ & \multirow{2}{*}{0.942} \\
\hline Neuropathy present & 17 & 7 & $41.1 \%$ & \\
\hline No neuropathy & 83 & 27 & $32.5 \%$ & \\
\hline Total & 100 & 34 & $34 \%$ & \\
\hline
\end{tabular}

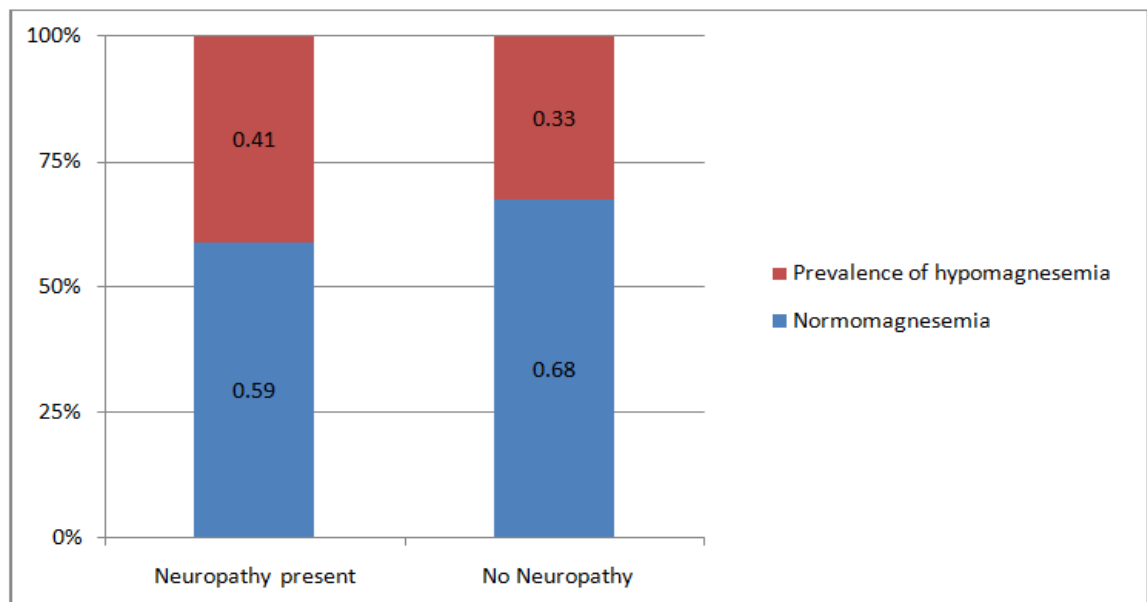




\section{Discussion}

The study population were grouped with regard to their age ,gender,duration of diabetes ,glycemic control and presence or absence of diabetic complication The study population comprised of 100 cases of type 2 diabetes mellitus

\section{Prevalence of hypomagnesemia}

The prevalence of hypomagnesemia in our study was $34 \%$, which was in concordance with the studies done by Rude RK et al.(3) in which prevalence of hypomagnesemia was 25-39\%.This was also in concordance with the study conducted by Walty MK et al.(5) and by Mohan K et al.(6) which showed prevalence of hypomagnesemia in $37.6 \%$ and $35 \%$ of the type 2 diabetes mellitus patients respectively .

\begin{tabular}{|l|l|l|l|l|}
\hline Study & Rude RK et al & Walti MK et al & Mohan K et al & Present study \\
\hline Hypomagnesemia(\%) & $25-39 \%$ & $37.6 \%$ & $35 \%$ & $34 \%$ \\
\hline
\end{tabular}

The variation in hypomagnesemia in different study group might be due to the difference in the definition of hypomagnesemia, techniques in the serum magnesium measurements and the heterogeneity of the selected patients groups. In our study possible causes of hypomagnesemia in the patients with type 2 diabetes mellitus may have been poor oral intake,poor gastrointestinal absorption due to diabetic autonomic gastropathy and also May be renal causes as in the patients with diabetes mellitus, the ultrafiltrable magnesium load may be enhanced by glomerular hyperfiltration, recurrent excessive volume repletion after hyperglycemia induced osmotic diuresis,recurrent metabolic acidosis associated with diabetic ketoacidosis and hypoalbuminemia.

\section{Hypomagnesemia according to patient's age,sex and duration of diabetes:}

In our study,hypomagnesemia was more prevalent in females(40\%),compared to males(30\%).This was in accordance with studies conducted by Ascaso J F et al. (7) and Sheehan J P et al.(8)have also reported a higher prevalence of hypomagnesemia in women compared to men.Our study had shown that higher prevalence $(40.7 \%)$ of hypomagnesemia in group with $\mathrm{HbA1} 1 \mathrm{c}>10$ with $\mathrm{p}$ value $=0.79$. another studies concludes plasma levels of magnesium have a strong significant negative correlation with HbA1c levels in diabetic group. $(9,10)$.

\section{Hypomagnesemia and diabetic complications:}

Previously magnesium deficiency has been found to be associated with diabetic microvascular disease.In the present study too significantly higher prevalence of hypomagnesemia was observed in diabetics with microvascular complications nad serum magnesium levels in diabetics with microvascular complications was comparatively lower than in diabetics with no microvascular complications. $(11,12)$

Hypomagnesemia was more pronounced in case of advanced retinopathy.Hypomagnesemia seem to be as possible risk factor in development and progress of diabetic retinopathy. In our observation,56.2\% patients with diabetic retinopathy were having low magnesium levels as compared to $23.5 \%$ patients without diabetic retinopathy and this difference was statistically significant $(\mathrm{p}$ value $=0.002)$

A significant difference was found between patients with nephropathy and no nephropathy,there was higher prevalence of hypomagnesemia in patients with nephropathy.In our study ,11 patients had microalbuminuria and 3 patients had macroalbuminuria.

In summary, the present study has demonstrated that hypomagnesemia is common in patients of Type 2 diabetes mellitus and magnesium deficiency is conclusively associated with diabetic retinopathy and diabetic nephropathy, so it may be prudent in clinical practice to periodically monitor serum magnesium levels in diabetic patients.If plasma nmagnesium level is low, an intervention $t$ increase dietary intakes of magnesium may be beneficial .However, the role of magnesium supplements remains to be evaluated.

\section{References}

[1]. American diabetic association.Diagnosis and classification of diabetes mellitus. Diabetic care.2012 Jan;s64-s71.

[2]. Barbagallo M, Domingurz LJ, Galioto A, Cani C,Malfa Let al.Role of magnesium in insulin action, diabetes and cardio-metabolic syndrome X. Mol Aspects Med.2003 Feb-Jun;24(1-3):39-52.

[3]. Rude RK. Magnesium deficiency and diabetes mellitus-cause and effects. Postgrad Med J.1992 Oct;92(5):217-24.

[4]. Pham PC, Pham PM, Pham SV, Miller JM, Pham PT. Hypomagnesemia in patients with type 2 diabetes.Clin J AM Soc Nephrol.2007 Mar;2(2):366-373.

[5]. Walti MK, Zimmermann MB, Spinas GA, Hurrell RF.Low plasma magnesium in type 2 diabetes.Swiss Med Wkly.2003 May;133(19-20):289-92

[6]. Antin SS, Kashinkunti M ,Kataria AV, Dhananjaya M, Alevoor S.A cross sectional study of fasting serum magnesium levels in the patients with type 2 diabetes and its relation to diabetic complications.Sch.J.of App.Med.Sci.2014;2(2A):502-506.

[7]. Ascaso J, Romero P, Real JT, Lorente RI, Martinez-Valls J,Carmena R.Abdominal obesity ,insulin resistance and metabolic syndrome in southern European population.Eur J Intern Med.2003 Mar;14(2):101-6

[8]. Sheehan JP.Magnesium deficiency and diabetes mellitus.Magnes Trace Elem.1991;10(2-4:215-219.

[9]. ShaikhMK,Devrajani BR, Soomro AA, Shah SZA, Devrajani T, Das T.Hypomagnesemia in patients with diabetes mellitus.World applied Sciences Journal.2011;12(10):1803-1806. 
[10]. Seedahmed TA, Ahmed SM.Correlation between the plasma magnesium levels and glycated haemoglobin in Sudanese patients with type 2 diabetes mellitus in Khoratum state.Advances in Diabetes and Metabolism.2013;1(1):1-5.

[11]. Kundu D, Osta M, Mandal T, Bandhopadhyay U, Ray D, Gautam D.Serum magnesium levels in patients with diabetic retinopathy. J Nat Sci Biol Med.2013 Jan;4(1):113-6.

[12]. Corsonello A, Lentile R, Buemi M, Cucinotta D, Mauro VN, Macaione S, et al.Serum ionized magnesium level in type 2 diabetic patients with microalbuminuria or clinical proteinuria. Am J Nephrol.2000 May-Jun;20(3):187-92. 